\title{
Shape controlled interpolatory ternary subdivision
}

\author{
C. Beccari ${ }^{\mathrm{a}, *}$, G. Casciola $^{\mathrm{a}}$, L. Romani ${ }^{\mathrm{b}}$ \\ ${ }^{a}$ Department of Mathematics, University of Bologna, \\ P.zza di Porta San Donato 5, 40127 Bologna, Italy \\ ${ }^{\mathrm{b}}$ Department of Mathematics and Applications, University of Milano-Bicocca, \\ Via R. Cozzi 53, 20125 Milano, Italy
}

\begin{abstract}
Ternary subdivision schemes compare favorably with their binary analogues because they are able to generate limit functions with the same (or higher) smoothness but smaller support.

In this work we consider the two issues of local tension control and conics reproduction in univariate interpolating ternary refinements. We show that both these features can be included in a unique interpolating 4-point subdivision method by means of non-stationary insertion rules that do not affect the improved smoothness and locality of ternary schemes. This is realized by exploiting local shape parameters associated with the initial polyline edges.
\end{abstract}

Key words: Univariate ternary refinement; Interpolation; Non-stationarity; Tension control; Conic section; Combined subdivision scheme

\section{Introduction}

The interest in investigating arities higher than two has started from the seminal paper by Hassan et al. [1]. Here they showed that, for the so-called interpolating 4-point stationary scheme, higher smoothness and smaller support can be achieved by going from binary to ternary.

At each iteration $k \geqslant 0$, an interpolating 4-point ternary subdivision scheme maps

* Corresponding author.

Email addresses: beccari@dm.unibo.it (C. Beccari), casciola@dm.unibo.it

(G. Casciola), lucia.romani@unimib.it (L. Romani). 
the polygon $P^{k}=\left\{p_{j}^{k}\right\}_{j \in \mathbb{Z}}$ to the refined polygon $P^{k+1}=\left\{p_{j}^{k+1}\right\}_{j \in \mathbb{Z}}$ through the insertion rules

$$
\begin{aligned}
p_{3 j}^{k+1} & =p_{j}^{k}, \\
p_{3 j+1}^{k+1} & =a_{0}^{k} p_{j-1}^{k}+a_{1}^{k} p_{j}^{k}+a_{2}^{k} p_{j+1}^{k}+a_{3}^{k} p_{j+2}^{k}, \\
p_{3 j+2}^{k+1} & =a_{3}^{k} p_{j-1}^{k}+a_{2}^{k} p_{j}^{k}+a_{1}^{k} p_{j+1}^{k}+a_{0}^{k} p_{j+2}^{k},
\end{aligned}
$$

where the coefficients $\left\{a_{i}^{k}\right\}_{i=0,1,2,3}$ are chosen in order to satisfy the relation

$$
a_{0}^{k}+a_{1}^{k}+a_{2}^{k}+a_{3}^{k}=1
$$

In general, the coefficients may either stay constant throughout the subdivision process or change according to the refinement level $k$. The scheme is said stationary in the first case and non-stationary in the latter.

Although Hassan's stationary refinement still looks like the most appealing proposal of interpolatory ternary subdivision, it does not provide great design flexibility. In fact, once the initial control points are chosen, this scheme does not allow the user to control the shape of the interpolant. Moreover, although such a scheme reproduces cubic polynomials starting from uniformly spaced initial samples, it does not reproduce any other analytic curve.

The goal of this paper is to improve the design performance of the 4-point ternary interpolating scheme, without affecting its improved smoothness and local support. We will thus consider two kinds of shape manipulations: one for controlling the tension of the limit curve in correspondence to each edge of the initial polygon and another for exactly reproducing circles and more generally conic sections. The first has a two-fold advantage: it allows us to arbitrarily modify the shape of the interpolant and, if the initial tension values are set properly, it may alleviate the undesired undulations that often arise as a consequence of the interpolation process. The second one, i.e. the capability of reproducing conic sections, is obviously a fundamental feature in many application contexts.

Our aim is defining an interpolating algorithm that reproduces conic sections in those regions of the initial polyline where the given samples belong to one of these curves and locally-controlled $C^{2}$ curves otherwise.

The definition of a locally-controlled interpolating ternary subdivision scheme has been already addressed in [2]. In that work, a tension parameter is associated with each edge of the initial polyline. In this way, by progressively increasing/decreasing its value, in the corresponding region of interest the limit curve tends to become tighter/looser to the underlying data polygon. After one initial parameter has been assigned to each edge, its value is automatically updated at each iteration by means of a recurrence formula. The corresponding refinement algorithm is non-stationary and generates $C^{2}$-continuous limit curves for any choice of the initial tensions in a wide span of definition. However it does not reproduce the whole class of conic sections nor any other analytic curve except quadratic polynomials. 
In binary subdivision literature, the construction of subdivision schemes reproducing conic sections has been addressed extensively [3-10]. Conversely, in the ternary context, there is still no available scheme for exactly generating these families of curves. The only attempt towards the definition of a ternary circle-preserving subdivision scheme has appeared in [11]. In that work, exploiting a refinement which preserves the discrete curvature and tangent direction at each vertex of the starting control polygon, a satisfactory approximation of a circle is generated starting from a regular $\kappa$-gon. However, the derived algorithm is quite involved, computationally heavy and, as acknowledged by the authors themselves, the smoothness of the related limit curves is also not known.

Thus, to the aim of exactly generating conic sections, we first address the construction of a non-stationary interpolating 4-point ternary refinement where the insertion rules are determined through Lagrange interpolation by functions from the 4-dimensional space $\left\{1, x, e^{t x}, e^{-t x}\right\}$, where $t \in\{0, s$, is $\mid s>0\}$. As a consequence of its definition, such a scheme reproduces certain exponential functions and in particular circles and conic sections (whenever the initial points are sampled at equally-spaced values of $t$ on these kinds of curves and the starting parameters are initialized accordingly). Similarly to the ternary 4-point scheme presented in [2], this refinement is non-stationary and its level-dependent coefficients are automatically computed by means of a recurrence formula based on a third-angle relation linking parameter values at two successive subdivision steps. We show that the proposed scheme generates $C^{1}$-continuous limit curves and we discuss how its property of reproducing conic sections depends on an opportune choice of the initial parameter. We finally present the unified subdivision scheme that combines the locally-controlled $C^{2}$ scheme in [2] with the derived exponentials reproducing scheme. The proposed algorithm is realized by allowing different regions of the initial polyline to be refined with one subdivision scheme or the other. Provided that the initial parameters are set properly, the combined scheme defined in this way generates arcs of conic sections where needed and locally-controlled $C^{2}$ curves otherwise. Moreover, in the region common to both schemes, its limit curves are $C^{1}$-continuous.

The paper is organized as follows. In Section 2 we start out by reviewing the locallycontrolled $C^{2}$ subdivision scheme presented in [2]. In Section 3, we define the novel ternary scheme reproducing exponentials (Section 3.1), analyze its smoothness (Section 3.2) and discuss how its parameter needs to be initialized according to the spacing of the given samples (Section 3.3). In Section 4 we finally present the refinement algorithm that unifies the two schemes described in Sections 2 and 3. Then we analyze its smoothness and illustrate some application examples.

\section{A locally-controlled $C^{2}$ interpolating 4-point ternary subdivision scheme}

The scheme in [2] is summarized by the following procedure. At the first stage, the polyline $P^{0}=\left\{p_{j}^{0}\right\}_{j \in \mathbb{Z}}$ and an initial set of parameters $\left\{v_{j}^{0}\right\}_{j \in \mathbb{Z}}$ are given. The value 
$v_{j}^{0}$ is assigned to the edge $\overline{p_{j}^{0} p_{j+1}^{0}}$.

At the iteration $k \geqslant 0$, the coarse polyline $P^{k}$ is transformed into the refined polyline $P^{k+1}$ by applying the 4-point insertion rules

$$
\begin{aligned}
p_{3 j}^{k+1} & =p_{j}^{k} \\
p_{3 j+1}^{k+1} & =a_{0, j}^{k} p_{j-1}^{k}+a_{1, j}^{k} p_{j}^{k}+a_{2, j}^{k} p_{j+1}^{k}+a_{3, j}^{k} p_{j+2}^{k} \\
p_{3 j+2}^{k+1} & =a_{3, j}^{k} p_{j-1}^{k}+a_{2, j}^{k} p_{j}^{k}+a_{1, j}^{k} p_{j+1}^{k}+a_{0, j}^{k} p_{j+2}^{k}
\end{aligned}
$$

with coefficients

$$
\begin{array}{ll}
a_{0, j}^{k}=\frac{1}{60}\left(-90 u_{j}^{k+1}-1\right) & a_{1, j}^{k}=\frac{1}{60}\left(90 u_{j}^{k+1}+43\right) \\
a_{2, j}^{k}=\frac{1}{60}\left(90 u_{j}^{k+1}+17\right) & a_{3, j}^{k}=\frac{1}{60}\left(-90 u_{j}^{k+1}+1\right)
\end{array}
$$

where

$$
u_{j}^{k+1}=-\frac{1}{3\left(1-\left(v_{j}^{k+1}\right)^{2}\right)\left(1+v_{j}^{k+1}\right)}
$$

and

$$
v_{j}^{k+1}=\sqrt{2+v_{j}^{k}} .
$$

Equation (3) is analogous to (1), except that the coefficients $\left\{a_{i, j}^{k}\right\}_{i=0, \ldots, 3}$ enclose the subscript index $j$ to underline their dependence on the $j$-th polyline edge.

After each iteration, the parameters are reassigned to the refined edges according to the rule $v_{3 j}^{k+1}=v_{3 j+1}^{k+1}=v_{3 j+2}^{k+1}:=\sqrt{2+v_{j}^{k}}$.

In [2] it is shown that this scheme tends to become stationary in the limit, since $\lim _{k \rightarrow+\infty} v_{j}^{k}=2$. Moreover it is also proved that the described subdivision algorithm generates $C^{2}$-continuous limit curves for any $v_{j}^{0} \in[-2,+\infty) \backslash\{-1\}^{1}$.

The peculiarity of this method is that the parameters assigned to the edges have a local tension effect. More precisely, the higher the value of $v_{j}^{0}$, the tighter the limit curve will be to the edge $\overline{p_{j}^{0} p_{j+1}^{0}}$. In this sense we say that this scheme is locallycontrolled and we call the $v_{j}^{0}$-s tension parameters.

Notice also that, when $v_{j}^{0}=2$, relation (6) implies that the scheme is stationary in correspondence to the $j$-th initial edge. In this case, for all $k \geqslant 0$, the coefficients assume the scalar values

$$
a_{0, j}^{\infty}:=-\frac{13}{180}, \quad a_{1, j}^{\infty}:=\frac{139}{180}, \quad a_{2, j}^{\infty}:=\frac{61}{180}, \quad a_{3, j}^{\infty}:=-\frac{7}{180} .
$$

$\overline{1}$ The initial value $v_{j}^{0}=-1$ is excluded to avoid a zero denominator in (5). 
As pointed out in the Introduction, the considered scheme does not reproduce any analytic curve except quadratic polynomials. Therefore, in the following section, we will address the definition of a ternary 4-point scheme that is able to exactly represent a certain family of exponential functions, including circles and conic sections as a particular case.

\section{An interpolating 4-point ternary subdivision scheme reproducing ex- ponentials}

\subsection{Construction of the scheme}

In [3] a binary non-stationary interpolatory subdivision algorithm was designed by up-sampling from the function space $V_{t}=\left\{1, x, e^{t x}, e^{-t x}\right\}$, where $t \in\{0, s$, is $\mid s>$ $0\}$. In this way, the insertion rule naturally reproduces cubic polynomials when $t=0$, hyperbolic functions when $t=s$ and trigonometric functions when $t=\mathrm{i} s$, $s>0$. Although such a scheme is non-stationary, at each iteration the refinement coefficients are simply updated by means of a recurrence relation involving the bisection formula of the cosine and hyperbolic cosine functions.

To generalize this refinement to the ternary case, the first necessary non-trivial step is thus to find a recurrence relation for iterated trisections of a given argument.

Definition 1 For each subdivision level $k \geqslant 0$, let

$$
w^{k}:=e^{t_{k}}+e^{-t_{k}}
$$

where $t_{k}=t / 3^{k}$.

Proposition 2 (Third-angle formula) For any $k \geqslant 0$, the parameters $w^{k}$ and $w^{k+1}$ defined in (8) satisfy the recurrence

$$
w^{k+1}=\Re\left[\left(\frac{w^{k}+\sqrt{\left(w^{k}\right)^{2}-4}}{2}\right)^{\frac{1}{3}}+\left(\frac{w^{k}+\sqrt{\left(w^{k}\right)^{2}-4}}{2}\right)^{-\frac{1}{3}}\right] .
$$

PROOF. When $t=0, w^{k}=2 \forall k \geqslant 0$, and hence equation (9) is trivially verified. When $t=\mathrm{i} s, s \in(0, \pi)$, from the identity $4 \cos ^{3}\left(s_{k+1}\right)-3 \cos \left(s_{k+1}\right)=\cos \left(s_{k}\right)$ it turns out that

$$
\cos \left(s_{k+1}\right)=\frac{1}{2} \Re\left[\left(\cos \left(s_{k}\right)+\sqrt{\cos ^{2}\left(s_{k}\right)-1}\right)^{\frac{1}{3}}+\left(\cos \left(s_{k}\right)+\sqrt{\cos ^{2}\left(s_{k}\right)-1}\right)^{-\frac{1}{3}}\right],
$$


from which (9) can be easily obtained.

The case $t=s, s>0$, can be treated analogously to the previous one due to the identity $4 \cosh ^{3}\left(s_{k+1}\right)-3 \cosh \left(s_{k+1}\right)=\cosh \left(s_{k}\right)$, from which it follows

$$
\cosh \left(s_{k+1}\right)=\frac{1}{2} \Re\left[\left(\cosh \left(s_{k}\right)+\sqrt{\cosh ^{2}\left(s_{k}\right)-1}\right)^{\frac{1}{3}}+\left(\cosh \left(s_{k}\right)+\sqrt{\cosh ^{2}\left(s_{k}\right)-1}\right)^{-\frac{1}{3}}\right] .
$$

Furthermore, the following lemma holds.

Lemma 3 The parameter $w^{k}$ satisfies the equalities

$$
e^{t_{k}}=\frac{w^{k}+\sqrt{\left(w^{k}\right)^{2}-4}}{2} \text { and } e^{-t_{k}}=\frac{w^{k}-\sqrt{\left(w^{k}\right)^{2}-4}}{2} .
$$

PROOF. Let us denote $s_{k}:=\frac{s}{3^{k}}$. By simple algebra, it is straightforward to verify that, depending on the value of $t$, equation (8) can be written as follows:

1. $w^{k}=2$, when $t=0$;

2. $w^{k}=2 \cosh \left(s_{k}\right)$, when $t=s, s>0$;

3. $w^{k}=2 \cos \left(s_{k}\right)$, when $t=\mathrm{i} s, s>0$.

In case 1 , relations (10) are trivially verified. In cases 2 and 3, they can be derived from Euler identities.

Let $t_{k}:=\frac{t}{3^{k}}$. If we now interpolate the equally-spaced data $\left\{\left(3^{-k} h, p_{j+h}^{k}\right)\right\}_{h=-1,0,1,2}$ with a function of the form $\varphi(x)=c_{0}+c_{1} x+c_{2} e^{t x}+c_{3} e^{-t x} \in V_{t}$, the unknown coefficients of $\varphi(x)$ are found as the unique solution to the linear system of equations $p_{j+h}^{k}=\varphi\left(3^{-k} h\right), h=-1,0,1,2$, namely

$$
\begin{aligned}
c_{0} & =\frac{\left(e^{-t_{k}}+e^{t_{k}}\right) p_{j}^{k}-p_{j-1}^{k}-p_{j+1}^{k}}{e^{-t_{k}}+e^{t_{k}}-2} \\
c_{1} & =3^{k} \frac{\left(e^{-t_{k}}+e^{t_{k}}+1\right)\left(p_{j+1}^{k}-p_{j}^{k}\right)+p_{j-1}^{k}-p_{j+2}^{k}}{e^{-t_{k}}+e^{t_{k}}-2} \\
c_{2} & =\frac{\left(e^{-2 t_{k}}+2 e^{t_{k}}-3\right)\left(p_{j-1}^{k}-2 p_{j}^{k}+p_{j+1}^{k}\right)-\left(e^{-t_{k}}+e^{t_{k}}-2\right)\left(2 p_{j-1}^{k}-3 p_{j}^{k}+p_{j+2}^{k}\right)}{\left(e^{-t_{k}}-e^{t_{k}}\right)\left(e^{-t_{k}}+e^{t_{k}}-2\right)^{2}} \\
c_{3} & =\frac{\left(e^{-t_{k}}+e^{t_{k}}-2\right)\left(2 p_{j-1}^{k}-3 p_{j}^{k}+p_{j+2}^{k}\right)-\left(e^{2 t} k+2 e^{-t_{k}}-3\right)\left(p_{j-1}^{k}-2 p_{j}^{k}+p_{j+1}^{k}\right)}{\left(e^{-t_{k}}-e^{t_{k}}\right)\left(e^{\left.-t_{k}+e^{t} k-2\right)^{2}}\right.}
\end{aligned}
$$

and they turn out to be well-defined when $t \neq 0$ and, whenever $t=\mathrm{i} s$, for $s \in(0, \pi)$. In this way we get the following result.

Proposition 4 Given any initial parameter $w^{0} \in(-2,+\infty)$, let 


$$
\begin{aligned}
& a_{0}^{k}=\frac{1+2 w^{k+1}}{3\left(1-w^{k+1}\right)\left(1+w^{k+1}\right)^{3}}, \\
& a_{1}^{k}=\frac{\left(2+w^{k+1}\right)\left(1-2\left(w^{k+1}\right)^{3}\right)}{3\left(1-w^{k+1}\right)\left(1+w^{k+1}\right)^{3}}, \\
& a_{2}^{k}=\frac{2\left(-1+w^{k+1}\right)-\left(w^{k+1}\right)^{3}\left(2+w^{k+1}\right)}{3\left(1-w^{k+1}\right)\left(1+w^{k+1}\right)^{3}}, \\
& a_{3}^{k}=\frac{2+w^{k+1}}{3\left(1-w^{k+1}\right)\left(1+w^{k+1}\right)^{3}}
\end{aligned}
$$

where $w^{k+1}$ is recursively computed through (9) for all $k \geqslant 0$. Then the ternary interpolating 4-point scheme defined by equations (1) with $\left\{a_{i}^{k}\right\}_{i=0,1,2,3}$ in (12) reproduces functions from the space $V_{t}$ (whenever the initial data are sampled at equally-spaced values of $t$ from a function in $V_{t}$ ).

PROOF. By substituting (10) into equations (11), the coefficients $\left\{c_{i}\right\}_{i=0,1,2,3}$ can be rewritten in terms of the parameter $w^{k+1}$. To get the insertion rules, it is then sufficient to compute the values of the interpolating function $\varphi(x)$ at the grid points $\frac{1}{3^{k+1}}$ and $\frac{2}{3^{k+1}}$ respectively, and express the newly inserted vertices $p_{j+\frac{1}{3}}^{k}$ and $p_{j+\frac{2}{3}}^{k}$ as linear combinations of $p_{j-1}^{k}, p_{j}^{k}, p_{j+1}^{k}, p_{j+2}^{k}$ with coefficients depending on the parameter $w^{k+1}$. This gives us the set of coefficients in (12). Since relations (12) are well-defined for any $w^{1} \neq 1$, hence for any $w^{0} \neq-2$, the choice of the initial parameter value is conveniently bounded to the range $w^{0} \in(-2,+\infty)$.

For the sake of conciseness we will refer to the refinement algorithm with coefficients in (12) as exponentials reproducing scheme (notice however that only functions in $V_{t}$ can be represented exactly). The advantage of the proposed scheme is that the recurrence relation (9) makes $w^{k}$ independent of the values of $t_{k}$ which vary from step to step. In fact, once $w^{0}$ has been chosen, at all successive subdivision steps its value is simply updated through (9) and the refinement coefficients $\left\{a_{i}^{k}\right\}_{i=0,1,2,3}$ are recomputed by substituting it in (12).

Remark 5 The novel subdivision scheme reproduces cubic polynomials. This is an immediate consequence of the observation that, when $t=0$, the solutions of the differential equation $\phi^{(4)}(x)-t^{2} \phi^{(2)}(x)=0$ turn out to be cubic polynomials.

\subsection{Smoothness analysis}

For the subdivision scheme presented in the previous subsection, the coefficients $\left\{a_{i}^{k}\right\}_{i=0,1,2,3}$ in (12) may vary from one refinement level to another. Hence the scheme is non-stationary and its smoothness properties can be derived by asymptotical equivalence with the corresponding stationary scheme. Two subdivision schemes $S_{a^{k}}$ and $S_{a}$ are said asymptotically equivalent if $\sum_{k \in \mathbb{Z}^{+}}\left\|S_{a^{k}}-S_{a}\right\|_{\infty}<+\infty$. In 
particular, our analysis is based on the following generalization of Theorem 8 in [12] to ternary subdivision.

Theorem 6 Let $S_{a^{k}}$ and $S_{a}$ be two asymptotically equivalent subdivision schemes, with $S_{a^{k}}$ non-stationary and $S_{a}$ stationary. Suppose $S_{a^{k}}$ and $S_{a}$ have finite support of equal width. If $S_{a}$ is $C^{m}$ and

$$
\sum_{k \in \mathbb{Z}^{+}} 3^{m k}\left\|S_{a^{k}}-S_{a}\right\|_{\infty}<+\infty
$$

then $S_{a^{k}}$ is $C^{m}$ too.

In addition, to the aim of our analysis, we need some preliminary results.

Lemma 7 Given $w^{0} \in(-2,+\infty)$, the recurrence relation (9) satisfies

$$
\lim _{k \rightarrow+\infty} w^{k}=2
$$

PROOF. Recall that a monotonic and bounded sequence is always convergent. Moreover, if it is non decreasing and upper bounded, then it converges to its upper bound. In the same way, if it is non increasing and lower bounded, then it converges to its lower bound. In particular, for the sequence defined by (9) with $w^{0} \in(-2,+\infty)$, it holds:

- if $w^{0} \in(-2,2)$, then the sequence $\left\{w^{k}\right\}_{k \geqslant 1}$ is non decreasing, since $\cos \left(s_{k+1}\right)$ $>\cos \left(s_{k}\right) \forall s \in(0, \pi)$;

- if $w^{0}=2$, then the sequence $\left\{w^{k}\right\}_{k \geqslant 1}$ is stationary;

- if $w^{0} \in(2,+\infty)$, then the sequence $\left\{w^{k}\right\}_{k \geqslant 1}$ is non increasing, since $\cosh \left(s_{k+1}\right)$ $<\cosh \left(s_{k}\right) \forall s>0$.

Therefore, in all the three cases above, $w^{k}$ is convergent and converges to 2 . In fact, if $\ell:=\lim _{k \rightarrow+\infty} w^{k}$, we have

$$
\ell=\Re\left[\left(\frac{\ell+\sqrt{\ell^{2}-4}}{2}\right)^{\frac{1}{3}}+\left(\frac{\ell+\sqrt{\ell^{2}-4}}{2}\right)^{-\frac{1}{3}}\right],
$$

and solving with respect to $\ell$ we get $\ell=2$.

This lemma allows us to view the Dubuc-Deslauriers ternary 4-point scheme $[13,14]$ as the limit of our scheme when $k \rightarrow+\infty$. In fact, it holds

$$
a_{0}^{\infty}:=-\frac{5}{81}, \quad a_{1}^{\infty}:=\frac{20}{27}, \quad a_{2}^{\infty}:=\frac{10}{27}, \quad a_{3}^{\infty}:=-\frac{4}{81} .
$$

Moreover, the two following lemmas establish a useful bound on the rate of convergence. 
Lemma 8 Let $w^{0} \in(-2,+\infty) \backslash\{2\}$. Then the sequence $\left\{w^{k}\right\}_{k \geqslant 1}$ in (9) satisfies the bound

$$
\frac{2-w^{k+1}}{2-w^{k}}<\frac{1}{3}, \quad \forall k \geqslant 0
$$

PROOF. To prove this result we will consider two cases separately.

1. $w^{0} \in(-2,2)$

Hence $w^{k} \in(1,2) \forall k>0$, and there exists $s \in(0, \pi)$ such that $s_{k}=\operatorname{acos}\left(w^{k} / 2\right)$. Thus $e^{\mathrm{i} s_{k}}=\frac{w^{k}+\sqrt{\left(w^{k}\right)^{2}-4}}{2}, w^{k+1}=2 \cos \left(s_{k+1}\right)$ and it is straightforward to see the validity of (14).

2. $w^{0} \in(2,+\infty)$

Hence $w^{k} \in(2,+\infty) \forall k>0$, and there exists $s>0$ such that $s_{k}=\operatorname{acosh}\left(w^{k} / 2\right)$. Thus $e^{s_{k}}=\frac{w^{k}+\sqrt{\left(w^{k}\right)^{2}-4}}{2}, w^{k+1}=2 \cosh \left(s_{k+1}\right)$ and (14) follows immediately.

Analogously the following lemma is easily proved.

Lemma 9 Let $w^{0} \in(-2,2)$. Then the sequence $\left\{w^{k}\right\}_{k \geqslant 1}$ in (9) satisfies the bound

$$
\frac{1-w^{k}}{1-w^{k+1}}<1, \quad \forall k \geqslant 0
$$

PROOF. As observed in case 1 of Lemma $8, w^{k}=2 \cos \left(s_{k}\right) \forall k$. Hence (15) follows straightforwardly, since $\cos \left(s_{k}\right)<\cos \left(s_{k+1}\right)$ and $1-2 \cos \left(s_{k+1}\right)<0, \forall s \in(0, \pi)$.

The above results can now be exploited to prove the asymptotic equivalence of the novel scheme with the Dubuc-Deslauriers ternary 4-point scheme.

Proposition 10 The non-stationary interpolating 4-point ternary scheme with coefficients defined by (12) and updated through relation (9), generates $C^{1}$ limit curves for any choice of the parameter $w^{0} \in(-2,+\infty)$.

PROOF. Let us consider the mask of the subdivision scheme (1)-(12)

$$
a^{k}=\left[a_{3}^{k}, a_{0}^{k}, 0, a_{2}^{k}, a_{1}^{k}, 1, a_{1}^{k}, a_{2}^{k}, 0, a_{0}^{k}, a_{3}^{k}\right] .
$$

The related first divided difference mask is

$$
d_{(1)}^{k}=\frac{1}{3\left(1-w^{k+1}\right)\left(1+w^{k+1}\right)^{3}}\left[2+w^{k+1},-1+w^{k+1},-1-2 w^{k+1},\right.
$$




$$
\begin{gathered}
3 w^{k+1}-2\left(w^{k+1}\right)^{3}-\left(w^{k+1}\right)^{4}, 3-2\left(w^{k+1}\right)^{3}-\left(w^{k+1}\right)^{4}, \\
\left.3 w^{k+1}-2\left(w^{k+1}\right)^{3}-\left(w^{k+1}\right)^{4},-1-2 w^{k+1},-1+w^{k+1}, 2+w^{k+1}\right] .
\end{gathered}
$$

To prove the thesis we will now show that $d_{(1)}^{k}$ generates $C^{0}$ limit curves. As, by Lemma 7,

$$
d_{(1)}^{\infty}:=\lim _{k \rightarrow+\infty} d_{(1)}^{k}=\frac{1}{81}[-4,-1,5,26,27,26,5,-1,-4]
$$

hence $d_{(1)}^{\infty}$ coincides with the first divided difference mask of the Dubuc-Deslauriers stationary scheme defined by (13). Since this scheme is known to be $C^{1}[13,14]$, the scheme associated with $d_{(1)}^{\infty}$ will be $C^{0}$. Now, if

$$
\sum_{k=0}^{+\infty}\left\|d_{(1)}^{k}-d_{(1)}^{\infty}\right\|_{\infty}<+\infty
$$

the two difference schemes are asymptotically equivalent and by Theorem 6 the scheme associated with $d_{(1)}^{k}$ is $C^{0}$ too.

To conclude the proof, relation (18) can be easily verified by using simple algebra and taking into account the results in Lemmas 8 and 9 (see Appendix A for the details of this computation).

Remark 11 Notice that, if compared with its binary counterpart in [3], the proposed scheme has the same smoothness order, but a smaller support. In particular, while the support of the considered scheme has width $2 \frac{5}{3} \sum_{k=0}^{+\infty} \frac{1}{3^{k}}=5$ (see [15]), the support of the corresponding binary scheme has width 6 .

\subsection{The proper setting of the parameter $w^{0}$}

In the following we will see how the initial parameter $w^{0}$ needs to be set to achieve the reproduction properties of the exponentials reproducing scheme defined in Section 3.1.

Let $P^{0}=\left\{p_{j}^{0}\right\}_{j \in \mathbb{Z}}$ be a set of uniform samples of a function in $V_{0}, V_{s}$ or $V_{\mathrm{i} s}$. By construction of the subdivision scheme, it is clear that the value of $w^{0}$ identifies the space to which the limit function belongs. In particular,

- if $w^{0} \in(-2,2)$, then $w^{0}=2 \cos (s)$ for some $s \in(0, \pi)$, and the limit function belongs to $V_{\text {is }}$ with $s=\operatorname{acos}\left(w^{0} / 2\right)$;

- if $w^{0}=2$, then the limit function belongs to $V_{0}$;

- if $w^{0} \in(2,+\infty)$, then $w^{0}=2 \cosh (s)$ for some $s>0$, and the limit function belongs to $V_{s}$ with $s=\operatorname{acosh}\left(w^{0} / 2\right)$. 
As a consequence, if all the starting control points are sampled at equally spaced parameter values on a cubic polynomial, the limit curve obtained through the exponentials reproducing scheme with $w^{0}=2$ is the cubic polynomial itself.

In the same way, a regular $\kappa$-gon refined with initial parameter $w^{0}=2 \cos (2 \pi / \kappa)$ converges to the circle passing through its vertices (see Fig. 1).

In our context, we are particularly interested in the practical purpose of generating circles of a fixed radius or specified conic sections starting from a given set of sample points. Hence we will now discuss in more detail how the initial parameter $w^{0}$ needs to be set in order to reproduce conic sections through the interpolatory scheme in Section 3.1.

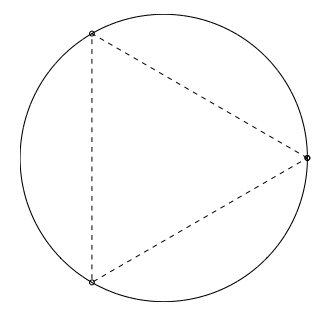

$w^{0}=-1$

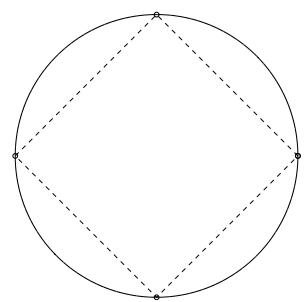

$w^{0}=0$

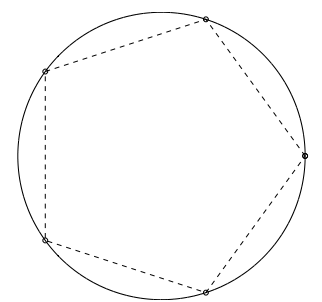

$w^{0}=2 \cos \left(\frac{2 \pi}{5}\right)$

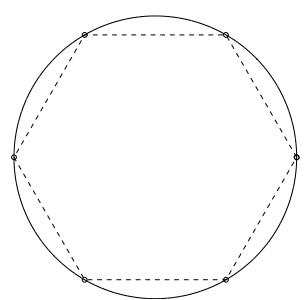

$w^{0}=1$

Fig. 1. Reproduction of circles through interpolation of regular $\kappa$-gons and related starting parameters.

Proposition 12 Let $w^{0}=2 \cosh (s u), s, u>0$. The subdivision scheme (1) with coefficients (12) reproduces the hyperbolic functions $f(x)=\cosh (s x)$ and $f(x)=$ $\sinh (s x)$ whenever the initial data points $\left\{\left(j u, p_{j}^{0}\right)\right\}_{j \in \mathbb{Z}}$ lie on such functions. In the same way, if $w^{0}=2 \cos (s u)$, with su $\in(0, \pi)$, the trigonometric functions $f(x)=\cos (s x)$ and $f(x)=\sin (s x)$ are exactly reproduced.

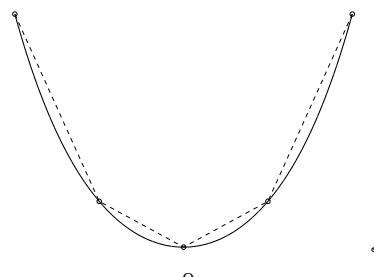

$w^{0}=2 \cosh (1)$

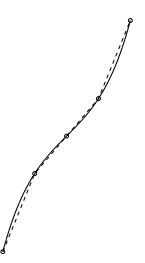

Fig. 2. Reproduction of hyperbolic and trigonometric functions starting from points

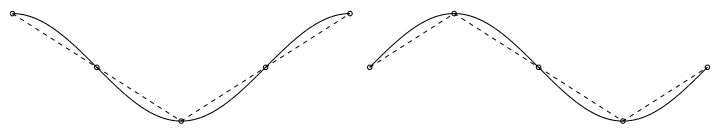

$w^{0}=0$ sampled with uniform parameter spacings $u=1$ and $u=\pi / 2$ respectively.

Corollary 13 Let $p_{j}^{0}=\{(a \cosh (j u), b \sinh (j u))\}_{j \in \mathbb{Z}}, u>0$, be equally spaced points on the parametric representation of the hyperbola. Then the limit curve obtained by choosing $w^{0}=2 \cosh (u)$ turns out to be the hyperbola itself (see Fig. 3 where $a=b=1, u=1)$.

The same holds if $p_{j}^{0}=\{(a \cos (j u), b \sin (j u))\}_{j \in \mathbb{Z}}, u \in(0, \pi)$, are on the ellipse with center 0 and radii $a, b$, and $w^{0}=2 \cos (u)$ (see Fig. 3 where $a=6, b=3, u=\frac{\pi}{2}$ ). 
In particular, when $a=b$, the limit curve is the circle of radius a (see Fig. 1 where $a=b=1$ and $u=\frac{2 \pi}{3}, \frac{\pi}{2}, \frac{2 \pi}{5}, \frac{\pi}{3}$ respectively).

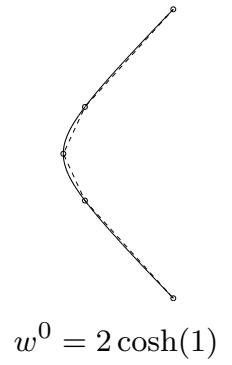

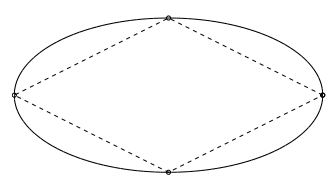

$w^{0}=0$

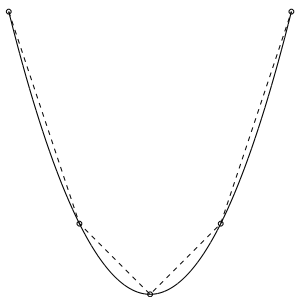

$w^{0}=2$

Fig. 3. Reproduction of conic sections starting from points sampled with uniform parameter spacings $u=1$ (hyperbola and parabola) and $u=\pi / 2$ (ellipse).

\section{The combined subdivision scheme}

In Section 2 we have reviewed a ternary interpolatory subdivision scheme with the properties of local tension control and $C^{2}$-smoothness. In Section 3 we have defined another ternary interpolatory scheme which reproduces conic sections, but, unlike the previous one, generates $C^{1}$ limit curves only. Both these schemes are described by interpolating ternary 4-point refinement equations.

Given an initial polyline, we consider now the problem of including in the related limit curve an alternation of pieces of conic sections in the regions where the starting samples belong to one of these curves and $C^{2}$ limit curves with local tension otherwise.

To this aim, we associate one of the two refinements in Sections 2 and 3 - either the locally controlled $C^{2}$ scheme or the exponentials reproducing scheme - with each edge of the initial polyline and we suppose that edge to be refined via the related scheme throughout all the subdivision process.

We will also use the following convention: any initial edge which has an associated parameter $v_{j}^{0}$ is refined through the locally-controlled $C^{2}$ scheme. Conversely, since the exponentials reproducing scheme has a global parameter, if the initial curve segment between the vertices $p_{j}^{0}$ and $p_{j+h+1}^{0}, h \in \mathbb{Z}^{+}$, corresponds to an arc of conic section, we denote its parameter with the symbol $w_{j, j+h}^{0}$ (see Fig. 4 (left)).

Remark 14 Once the starting values $v_{j}^{0}$ and $w_{j, j+h}^{0}$ have been chosen, at each iteration $k>0$ they are automatically updated and reassigned to the new edges by exploiting the respective recurrence formulas (6) and (9).

Basically, the proposed method consists in using two different refinements to the left and to the right hand side of a contact point, hence it belongs to the so-called piecewise uniform or combined subdivision schemes $[16,17]$. In particular, since all the schemes that are applied locally have bounded support, after enough iterations each 

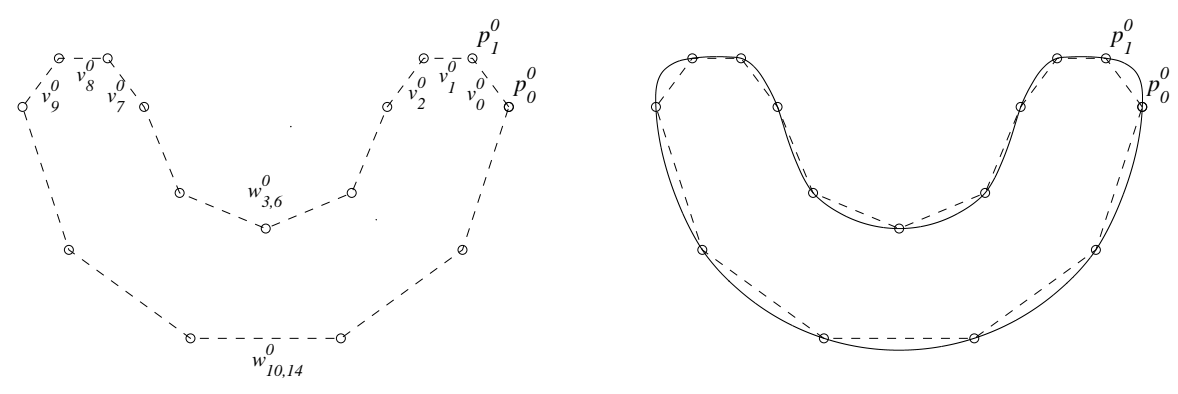

Fig. 4. Initial parameters setting in a polyline including an alternation of circular arcs and free-form curve segments (left); limit curve obtained via initial parameters $\left[v_{0}^{0}, v_{1}^{0}, v_{2}^{0}, w_{3,6}^{0}, v_{7}^{0}, v_{8}^{0}, v_{9}^{0}, w_{10,14}^{0}\right]=\left[1,10,1,2 \cos \left(\frac{\pi}{4}\right), 1,10,1,2 \cos \left(\frac{\pi}{5}\right)\right]$ (right).

contact point is affected only by the two schemes applied in its neighboring intervals. Hence, without loss of generality, we confine ourselves to analyze the configuration illustrated in Fig. 5. This situation corresponds to applying the locally-controlled $C^{2}$ scheme to the left of the contact point $\bar{p}$ and the exponentials reproducing scheme to the right. Thus we respectively denote these two schemes by $S_{l}^{k}$ and $S_{r}^{k}$ and the combined scheme by $S^{k}$.

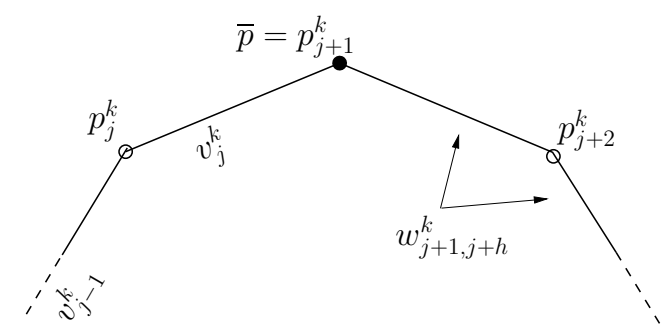

Fig. 5. Configuration of parameters around the contact point $\bar{p}$ at the $k$-th refinement step.

In the remainder of this section we are interested in determining the smoothness order of the limit curves generated by the proposed combined method.

The analysis of such a scheme reduces to the analysis of the two schemes $S_{l}^{k}$ and $S_{r}^{k}$ themselves and to the analysis of their behavior in the bounded region where they overlap. The two schemes $S_{l}^{k}$ and $S_{r}^{k}$ have been respectively analyzed in [2] and in Section 3.2. From this analysis we can say that, away from the contact point $\bar{p}$, the limit functions of $S^{k}$ are either $C^{2}$ or $C^{1}$, depending on whether they are generated by $S_{l}^{k}$ or $S_{r}^{k}$. Therefore we only need to study the regularity of the scheme $S^{k}$ in their overlapping region.

In a neighborhood of the contact point $\bar{p}, S^{k}$ is represented by the bi-infinite subdivision matrix 


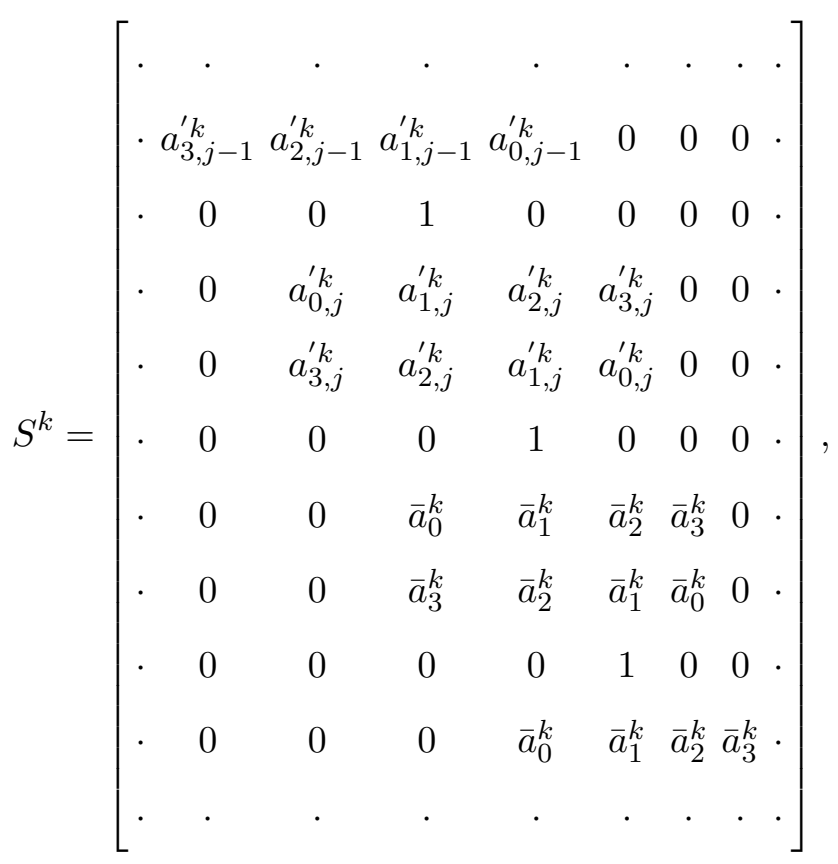

where $\left\{a_{i, j}^{\prime k}\right\}_{i=0, \ldots, 3}$ denote the coefficients (4) of the locally-controlled $C^{2}$ scheme and $\left\{\bar{a}_{i}^{k}\right\}_{i=0, \ldots, 3}$ the coefficients (12) of the exponentials reproducing scheme.

The resulting refinement is piecewise uniform and non-stationary at the same time, thus, for its analysis, we proceed as follows. First we determine the smoothness of the subdivision scheme obtained by replacing the elements of $S^{k}$ with their limit values $\left\{a_{i, j}^{\prime \infty}\right\}_{i=0, \ldots, 3}$ in $(7)$ and $\left\{\bar{a}_{i}^{\infty}\right\}_{i=0, \ldots, 3}$ in (13). We denote this limit scheme, which is still piecewise uniform but stationary, by $S^{\infty}$ (analogously the limit schemes of $S_{l}^{k}$ and $S_{r}^{k}$ are $S_{l}^{\infty}$ and $S_{r}^{\infty}$.) Successively we analyze $S^{k}$ by establishing its asymptotical equivalence to $S^{\infty}$.

Proposition 15 The subdivision scheme $S^{\infty}$ generates $C^{1}$-continuous limit curves in the region where $S_{l}^{\infty}$ and $S_{r}^{\infty}$ overlap.

PROOF. Generalizing Theorem 3 in [16] to ternary subdivision, it turns out that, in order to prove $C^{1}$-smoothness of the scheme $S^{\infty}$, it is sufficient to show that, in a proper $n \times n$ neighborhood of the contact point, the local subdivision matrix of $S^{\infty}$ has eigenvalues

$$
\lambda_{1}=1, \quad \lambda_{2}=\frac{1}{3}, \quad\left|\lambda_{i}\right|<\frac{1}{3}, \quad i=3, \ldots, n .
$$

In the following we verify that this condition holds for our subdivision matrix.

For ternary schemes, that are both primal and dual, the eigenvalues of the local subdivision matrix $S^{\infty}$ shall be worked out following the approach in [1]. Based on these results, we need to analyze the sub-matrices corresponding to points that are topologically invariant under the subdivision step [18]. After enough refinements, 
this implies analyzing the sub-matrix $S_{\bar{p}}^{\infty}$ corresponding to the neighborhood of the contact point $\bar{p}$ (everywhere else only the scheme $S_{l}^{\infty}$ or $S_{r}^{\infty}$ exists). Thus we have

$$
S_{\bar{p}}^{\infty}=\left[\begin{array}{ccccc}
a_{0, j}^{\prime \infty} & a_{1, j}^{\prime \infty} & a_{2, j}^{\prime \infty} & a_{3, j}^{\prime \infty} & 0 \\
a_{3, j}^{\prime \infty} & a_{2, j}^{\prime \infty} & a_{1, j}^{\prime \infty} & a_{0, j}^{\prime \infty} & 0 \\
0 & 0 & 1 & 0 & 0 \\
0 & \bar{a}_{0}^{\infty} & \bar{a}_{1}^{\infty} & \bar{a}_{2}^{\infty} & \bar{a}_{3}^{\infty} \\
0 & \bar{a}_{3}^{\infty} & \bar{a}_{2}^{\infty} & \bar{a}_{1}^{\infty} & \bar{a}_{0}^{\infty}
\end{array}\right]
$$

Substituting the limit values $\left\{a_{i, j}^{\prime \infty}\right\}_{i=0, \ldots, 3}$ and $\left\{\bar{a}_{i}^{\infty}\right\}_{i=0, \ldots, 3}$ respectively with $(7)$ and (13), we can easily work out the eigenvalues of $S_{\bar{p}}^{\infty}$. Since we get $\lambda_{1}=1, \lambda_{2}=$ $\frac{1}{3}, \lambda_{3}=0.1140, \lambda_{4}=\frac{1}{9}, \lambda_{5}=0.0168$, conditions (20) are all satisfied.

In particular, as a consequence of this last proposition, the difference scheme of $S^{\infty}$ is $C^{0}$.

Proposition 16 The subdivision scheme $S^{k}$ generates $C^{1}$-continuous limit curves in the region where $S_{l}^{k}$ and $S_{r}^{k}$ overlap.

PROOF. Let us denote the difference schemes of $S^{k}$ and $S^{\infty}$ by $D_{(1)}^{k}$ and $D_{(1)}^{\infty}$ respectively. Recalling Theorem 6 in Section 3.2, to prove our thesis, it is now sufficient to show that

$$
\sum_{k \in \mathbb{Z}^{+}}\left\|D_{(1)}^{k}-D_{(1)}^{\infty}\right\|_{\infty}<+\infty
$$

Substituting into (19) the expressions (4) and (12) it turns out that, in the neighborhood of the contact point $\bar{p}$, the difference matrix $D_{(1)}^{k}$ has the form

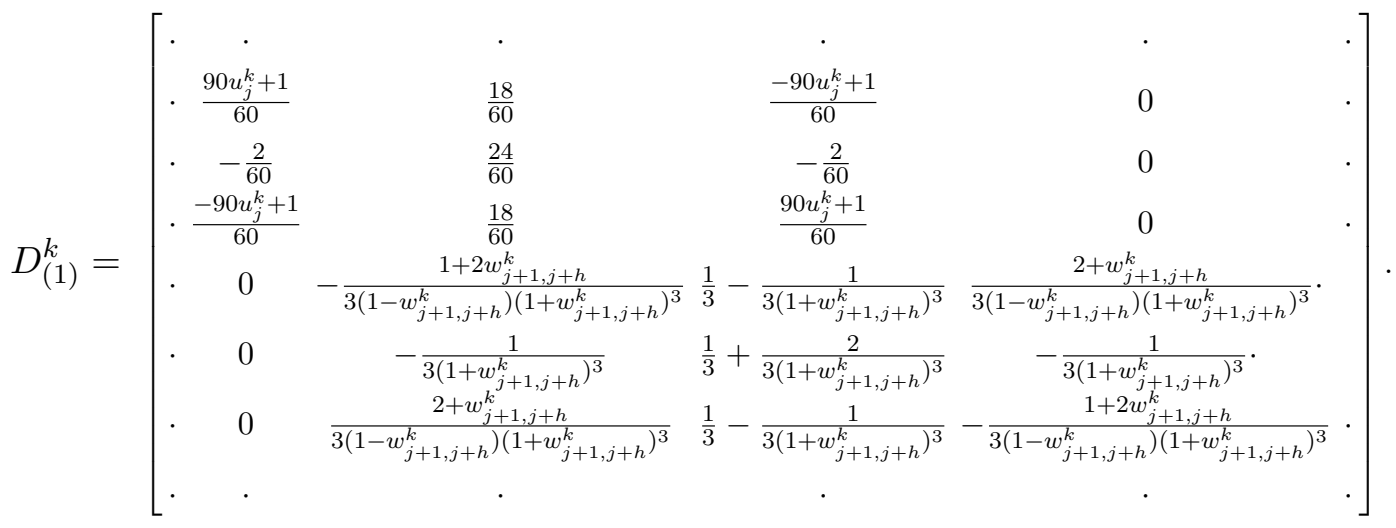


Clearly, $D_{(1)}^{\infty}$ is the matrix obtained from (22) by replacing $u_{j}^{k}$ and $w_{j+1, j+h}^{k}$ with their limit values. It can be easily observed that the rows of $D_{(1)}^{k}-D_{(1)}^{\infty}$ bring back either to the first divided difference of the scheme in Section 2 (see equation (16) in [2]) or to the first divided difference of the scheme in Section 3 (see equation (17)). As it was already proved, in both cases (21) is always verified. Hence the thesis follows straightforwardly.

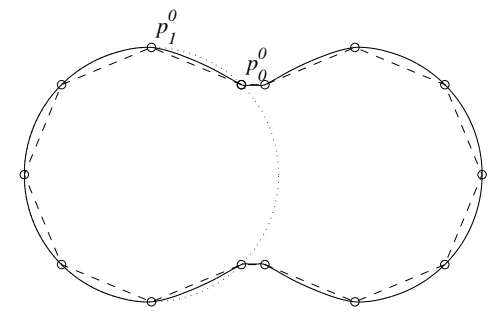

(a)

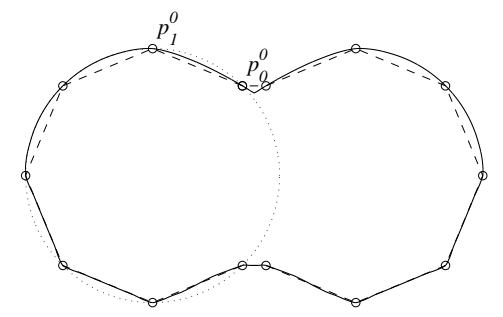

(c)

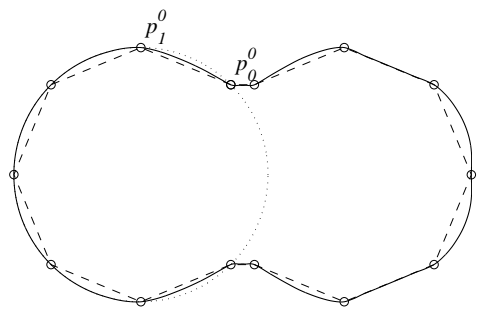

(b)

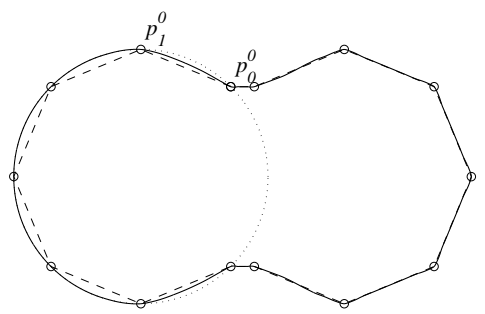

(d)

Fig. 6. Interpolation examples using the combined subdivision scheme.

In Figures 6 and 7 we illustrate how the proposed algorithm allows us to include in the same curve arcs of conic sections and free-form $C^{2}$ curve segments. The pictures in Fig. 6 correspond to the following choices of parameters
(a) $\left[w_{0,5}^{0}, v_{6}^{0}, w_{7,12}^{0}, v_{13}^{0}\right]=\left[2 \cos \left(\frac{\pi}{4}\right), 20,2 \cos \left(\frac{\pi}{4}\right), 20\right]$;
(b) $\left[w_{0,5}^{0}, v_{6}^{0}, v_{7}^{0}, v_{8}^{0}, v_{9}^{0}, v_{10}^{0}, v_{11}^{0}, v_{12}^{0}, v_{13}^{0}\right]=\left[2 \cos \left(\frac{\pi}{4}\right), 20,1,100,1,1,100,1,20\right]$;
(c) $\left[w_{0,2}^{0}, v_{3}^{0}, \ldots, v_{9}^{0}, w_{10,12}^{0}, v_{13}^{0}\right]=\left[2 \cos \left(\frac{\pi}{4}\right), 20, \ldots, 20,2 \cos \left(\frac{\pi}{4}\right), 1\right]$;
(d) $\left[w_{0,5}^{0}, v_{6}^{0}, v_{7}^{0}, v_{8}^{0}, \ldots, v_{11}^{0}, v_{12}^{0}, v_{13}^{0}\right]=\left[2 \cos \left(\frac{\pi}{4}\right), 10,10,100, \ldots, 100,10,10\right]$. 
The examples in Fig. 7 have been obtained by setting the parameters to the values

(a) $\left[v_{0}^{0}, v_{1}^{0}, w_{2,7}^{0}, v_{8}^{0}, v_{9}^{0}, v_{10}^{0}, v_{11}^{0}, w_{12,17}^{0}, v_{18}^{0}, v_{19}^{0}\right]=$ $\left[-0.5,1,2 \cos \left(\frac{\pi}{6}\right), 1,-0.5,1,1,2 \cos \left(\frac{\pi}{6}\right), 1,1\right] ;$

(b) $\left[v_{0}^{0}, v_{1}^{0}, w_{2,7}^{0}, v_{8}^{0}, v_{9}^{0}, v_{10}^{0}, v_{11}^{0}, w_{12,17}^{0}, v_{18}^{0}, v_{19}^{0}\right]=$ $\left[20,0.8,2 \cos \left(\frac{\pi}{6}\right), 0.8,20,-0.75,1,2 \cos \left(\frac{\pi}{6}\right), 1,-0.75\right]$;

(c) $\left[v_{0}^{0}, v_{1}^{0}, v_{2}^{0}, \ldots, v_{7}^{0}, v_{8}^{0}, v_{9}^{0}, v_{10}^{0}, v_{11}^{0}, w_{12,17}^{0}, v_{18}^{0}, v_{19}^{0}\right]=$ $\left[20,0.8,20, \ldots, 20,0.8,20,1,1,2 \cos \left(\frac{\pi}{6}\right), 1,1\right]$;

(d) $\left[v_{0}^{0}, v_{1}^{0}, w_{2,7}^{0}, v_{8}^{0}, v_{9}^{0}, v_{10}^{0}, v_{11}^{0}, w_{12,17}^{0}, v_{18}^{0}, v_{19}^{0}\right]=$ $\left[20,0.8,2 \cos \left(\frac{\pi}{6}\right), 0.8,20,30,1,2 \cos \left(\frac{\pi}{6}\right), 1,30\right]$.

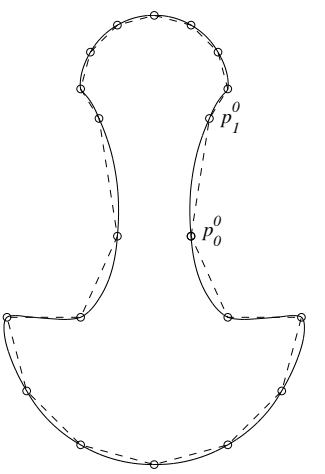

(a)

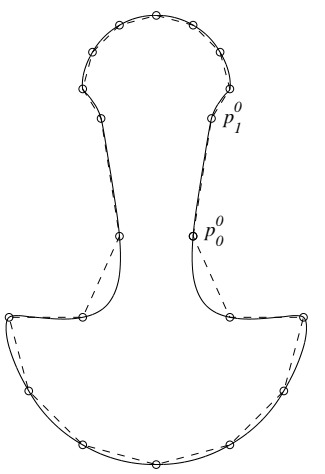

(b)

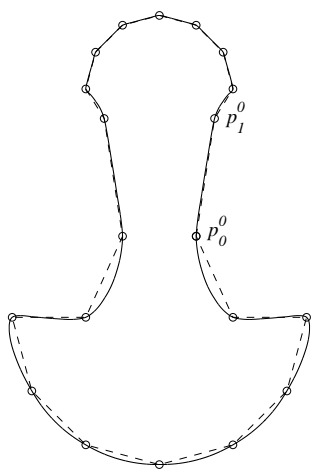

(c)

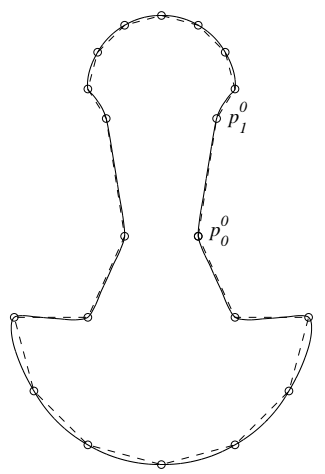

(d)

Fig. 7. Interpolation examples using the combined subdivision scheme.

We remark that the last one and a half spans at each end of the conic part of a curve which includes both schemes (see Figs. 6-7) do not converge to the conic. In fact they lie within the support of a control point which is not part of the conic formulation.

We finally observe that the proposed subdivision algorithm can be easily generalized to open polylines. To this aim, it is sufficient to uniformly extend the open control polygon by one additional vertex at the beginning and at the end. In particular, if the boundary vertices belong to a conic section, we extrapolate the additional vertex on that conic section, otherwise we derive it by linear extrapolation. (see Fig. 8). The examples in Fig. 8 have been obtained through the parameter values
(a) $\left[w_{0,6}^{0}, v_{7}^{0}, w_{8,10}^{0}\right]=\left[2 \cos \left(\frac{\pi}{4}\right), 2.1,2 \cos \left(\frac{\pi}{3}\right)\right]$;
(b) $\left[w_{0,6}^{0}, v_{7}^{0}, v_{8}^{0}, \ldots, v_{10}^{0}\right]=\left[2 \cos \left(\frac{\pi}{4}\right), 4,10, \ldots, 10\right]$. 


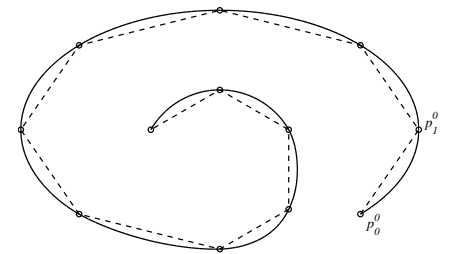

(a)

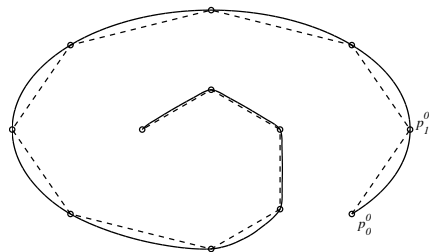

(b)

Fig. 8. Interpolation of an open polyline through the combined subdivision scheme.

\section{Conclusions}

To the aim of exactly reproducing conic sections, we have defined a novel nonstationary 4-point ternary refinement algorithm reproducing functions from the space $\left\{1, x, e^{t x}, e^{-t x}\right\}$ with $t=0, t=s$ or $t=\mathrm{i} s, s>0$. This scheme has the same smoothness, but smaller support, than its binary counterpart [3].

Successively, we have presented a unified formulation for the derived exponentials reproducing scheme and the locally-controlled $C^{2}$ subdivision scheme in [2]. The resulting ternary algorithm allows us to efficiently define limit curves that combine all the ingredients of locality, $C^{2}$ smoothness, user-independence, local tension control and reproduction of fundamental shapes.

\section{Appendix A: proof of relation (18)}

We prove here that the series in (18) is convergent for any $w^{0} \in(-2,+\infty)$. Since when $w^{0} \in(-2,2)$ then $w^{k+1} \in(1,2)$, and when $w^{0} \in(2,+\infty)$ then $w^{k+1} \in$ $(2,+\infty), \forall k \geqslant 0$, a straightforward calculation gives

$$
\left\|d_{(1)}^{k}-d_{(1)}^{\infty}\right\|_{\infty}=\frac{2\left|w^{k+1}-2\right|\left[5\left(w^{k+1}\right)^{3}+20\left(w^{k+1}\right)^{2}+40 w^{k+1}+16\right]}{81\left(w^{k+1}-1\right)\left(1+w^{k+1}\right)^{3}} .
$$

We will now consider two cases separately.

1. $w^{0} \in(-2,2)$

$$
\left\|d_{(1)}^{k}-d_{(1)}^{\infty}\right\|_{\infty}=\frac{2\left(2-w^{k+1}\right)\left[5\left(w^{k+1}\right)^{3}+20\left(w^{k+1}\right)^{2}+40 w^{k+1}+16\right]}{81\left(w^{k+1}-1\right)\left(1+w^{k+1}\right)^{3}}
$$

with

$$
\frac{5\left(w^{k+1}\right)^{3}+20\left(w^{k+1}\right)^{2}+40 w^{k+1}+16}{\left(1+w^{k+1}\right)^{3}}<\frac{81}{8} .
$$


Then

$$
\sum_{k=0}^{+\infty}\left\|d_{(1)}^{k}-d_{(1)}^{\infty}\right\|_{\infty}<\frac{1}{4} \sum_{k=0}^{+\infty} \frac{2-w^{k+1}}{w^{k+1}-1}
$$

Now, since $\frac{2-w^{k+1}}{w^{k+1}-1}>0$, convergence of this series can be proved by the ratio test and by exploiting Lemmas 8 and 9 . In particular it results

$$
\frac{\frac{2-w^{k+1}}{w^{k+1}-1}}{\frac{2-w^{k}}{w^{k}-1}}=\frac{2-w^{k+1}}{2-w^{k}} \frac{w^{k}-1}{w^{k+1}-1}<\frac{1}{3} 1<1 .
$$

2. $w^{0} \in(2,+\infty)$

$\left\|d_{(1)}^{k}-d_{(1)}^{\infty}\right\|_{\infty}=\frac{2\left(w^{k+1}-2\right)\left[5\left(w^{k+1}\right)^{3}+20\left(w^{k+1}\right)^{2}+40 w^{k+1}+16\right]}{81\left(w^{k+1}-1\right)\left(1+w^{k+1}\right)^{3}}$

with

$$
\frac{5\left(w^{k+1}\right)^{3}+20\left(w^{k+1}\right)^{2}+40 w^{k+1}+16}{\left(w^{k+1}-1\right)\left(1+w^{k+1}\right)^{3}}<8 .
$$

Then

$$
\sum_{k=0}^{+\infty}\left\|d_{(1)}^{k}-d_{(1)}^{\infty}\right\|_{\infty}<\frac{16}{81} \sum_{k=0}^{+\infty}\left(w^{k+1}-2\right)
$$

and, due to Lemma 8, convergence is proven by the ratio test. In particular it results

$$
\frac{w^{k+1}-2}{w^{k}-2}<\frac{1}{3}<1
$$

By unifying the cases 1 and 2 above, relation (18) is thus verified for any choice of the initial parameter $w^{0} \in(-2,+\infty)$.

\section{Acknowledgements}

This research was supported by the University of Bologna and the University of Milano-Bicocca, Italy.

\section{References}

[1] M.F. Hassan, I.P. Ivrissimtzis, N.A.Dodgson, M.A. Sabin, An interpolating 4point ternary stationary subdivision scheme. Comput. Aided Geom. Design 
19:1-18 (2002).

[2] C. Beccari, G. Casciola, L. Romani, An interpolating 4-point $C^{2}$ ternary nonstationary subdivision scheme with tension control. Comput. Aided Geom. Design 24:210-219 (2007).

[3] C. Beccari, G. Casciola, L. Romani, A non-stationary uniform tension controlled interpolating 4-point scheme reproducing conics. Comput. Aided Geom. Design 24:1-9 (2007).

[4] K. Beets, J. Claes, F. Van Reeth, A subdivision scheme to model surfaces with spherelike features. WSCG'2005 Conference Proceedings, 103-108 (2005).

[5] P. Chalmovianský, B. Jüttler, A non-linear circle-preserving subdivision scheme. Adv. Comput. Math. 27:375-400 (2007).

[6] N. Dyn, D. Levin, A. Luzzatto, Exponentials reproducing subdivision schemes. Found. Comput. Math 3:187-206 (2003).

[7] G. Morin, J. Warren, H. Weimer, A subdivision scheme for surfaces of revolution. Comput. Aided Geom. Design 18:483-502 (2001).

[8] M.A. Sabin, N.A. Dodgson, A circle-preserving variant of the four-point subdivision scheme, in Mathematical Methods for Curves and Surfaces: Troms $\varnothing$ 2004 (M. Dæhlen, K. Mørken and L.L. Schumaker, Eds.), Nashboro Press, 2005.

[9] J. Zhang, F.-L. Krause, Extending cubic uniform B-splines by unified trigonometric and hyperbolic basis. Graphical Models 67:100-119 (2005).

[10] J. Zhang, F.-L. Krause, H. Zhang, Unifying C-curves and H-curves by extending the calculation to complex numbers. Comput. Aided Geom. Design 22:865-883 (2005).

[11] M. Jeon, D. Han, K. Park, G. Choi, Ternary univariate curvature-preserving subdivision. J. of Appl. Math. \& Computing 18:235-246 (2005).

[12] N. Dyn, D. Levin, Analysis of asymptotically equivalent binary subdivision schemes. J. Math. Anal. Appl. 193:594-621 (1995).

[13] G. Deslauriers, S. Dubuc, Symmetric iterative interpolation processes. Constr. Approx. 5:49-68 (1989).

[14] S. Dubuc, Interpolation through an iterative scheme. J. Math. Anal. Appl. 114:185-204 (1986).

[15] A. Cohen, N. Dyn, Nonstationary subdivision schemes and multiresolution analysis. SIAM J. on Math. Anal. 27:1745-1769 (1996).

[16] N. Dyn, J.A. Greogory, D. Levin, Piecewise uniform subdivision schemes, in Mathematical Methods for Curves and Surfaces (M. Dæhlen, T. Lyche and L.L. Schumaker, Eds.), Vanderbilt University Press, 1995.

[17] A. Levin, Combined Subdivision Schemes. PhD thesis, Tel-Aviv University, 2000 . 
[18] M.A. Sabin, Eigenanalysis and artifacts of subdivision curves and surfaces, in Tutorials on multiresolution in geometric modelling (A. Iske, E. Quak, M.S. Floater, Eds.), Springer, 2002. 